\title{
Efficient Algorithm On a Non-staggered Mesh for Simulating Rayleigh-Bénard Convection in a Box
}

\author{
K.-H. Chiam \\ Nonlinear and Statistical Physics, California Institute of Technology, Mail Code 114-36, \\ Pasadena, CA 91125-3600 \\ Ming-Chih Lai \\ Department of Applied Mathematics, National Chiao Tung University, Hsinchu 300, Taiwan \\ Henry S. Greenside*† \\ Department of Physics, Duke University, P. O. Box 90305, Durham, NC 27708-0305
}

(October 29, 2018)

\begin{abstract}
An efficient semi-implicit second-order-accurate finite-difference method is described for studying incompressible Rayleigh-Bénard convection in a box, with sidewalls that are periodic, thermally insulated, or thermally conducting. Operator-splitting and a projection method reduce the algorithm at each time step to the solution of four Helmholtz equations and one Poisson equation, and these are are solved by fast direct methods. The method is numerically stable even though all field values are placed on a single non-staggered mesh commensurate with the boundaries. The efficiency and accuracy of the method are characterized for several representative convection problems.
\end{abstract}

Typeset using REVTEX 


\section{INTRODUCTION}

Experiments over the last three decades have discovered many fascinating and poorly understood examples of pattern formation in large-aspect-ratio Rayleigh-Bénard convection $[1,2]$. Because of the prominent role that these experiments play in understanding sustained nonequilibrium systems [2] and because many of the observed phenomena such as spatiotemporal chaos are difficult to analyze mathematically [3], there is a need to develop computer codes that can simulate these experiments quantitatively so that theory and experiment can be compared with one another. Once validated, such codes can further be used to explore regimes not easily attained by experiment such as low Prandtl number, and to calculate quantities that are difficult to deduce from experimental data, such as mean flows $[4,5]$ and fractal dimensions $[6]$.

The regime of large aspect ratio $\Gamma$ (ratio of horizontal fluid width to fluid depth) poses significant computational challenges. Many numerical degrees of freedom (basis functions or mesh points) are needed to represent the spatial features of the fluid and often the dynamics needs to be studied over long times (many multiples of the horizontal thermal diffusion time $\tau_{h}=\Gamma^{2} \tau_{v}$, where $\tau_{v}=d^{2} / \kappa$ is the vertical thermal diffusion time defined in terms of the fluid depth $d$ and fluid thermal diffusivity $\kappa$ ) to insure that nontransient behavior is being observed. Since the largest time step allowed by numerical stability for explicit or semi-implicit algorithms (the ones most commonly used in Navier-Stokes calculations) is typically $0.05 \tau_{v}$ or smaller, simulations in a representative $\Gamma=50$ cell may require $10^{5}$ or more time steps to eliminate a transient and then study the statistically stationary properties of the asymptotic dynamics. The many degrees of freedom and long integration times together with the need to repeat runs for different parameter values imply that efficient algorithms are essential for studying the large-aspect-ratio regime.

Because of these computational challenges, there have been few simulations of threedimensional Rayleigh-Bénard convection with aspect ratios exceeding 10. Recent calculations with $\Gamma$ as large as 64 have been carried out by Pesch and collaborators, who used a 
pseudospectral code on serial and parallel computers to study spiral defect chaos, rotating convection, and other problems [6-8]. However, their code uses periodic boundaries and so can not take into account quantitatively the influence of lateral walls on the bulk dynamics. Arter and Newell [9] and Tomita and Abe [10] have carried out simulations in large boxes with thermally insulated no-slip sidewalls, the former in a $16 \times 11.5$ aspect ratio box, the latter in a $\Gamma=18.84$ square box. Xi et al [11] have studied the transition to spatiotemporal chaos of a convecting fluid in a $\Gamma=60$ square cell, but with free-slip horizontal boundaries that are difficult to achieve experimentally. Finally, a Caltech-Duke collaboration has recently reported results $[12,13]$ obtained with a parallel spectral element code [14] for aspect ratios up to 30. Their code can treat quantitatively most geometries and lateral boundaries used by experimentalists, including ramps [15], spoiler fins [16], and lateral walls of finite thickness and finite thermal conductivity. However, the generality of the spectral element algorithm makes it substantially more expensive to run than algorithms optimized for a simple geometry such as a box or cylinder.

In this paper, we introduce and analyze an efficient semi-implicit finite-difference algorithm for studying incompressible Rayleigh-Bénard convection in a box, with lateral walls that are periodic, thermally insulated, or thermally conducting. The code complements the more flexible spectral element approach $[12,13]$ by being more than an order of magnitude more efficient on a serial processor, for a box with these boundary conditions. It is well suited for studying long-time dynamics of small- to moderate-aspect-ratio boxes [4] $(\Gamma \leq 20)$, with lateral boundaries that are close to those of many experiments, although not fully quantitatively accurate since the finite thickness and finite thermal diffusivity of the lateral walls is not taken into account.

The main advantages of our algorithm are the simplicity of implementation and its efficiency on a single processor. Its simplicity arises from the use of a single mesh for all field values. (This is called a "non-staggered" or "collocated" mesh in contrast to a "staggered" mesh for which the values of different fields appear at different points in space [17-19]). A non-staggered mesh reduces the effort to write and to validate a code (compared to one 
using a staggered mesh), and facilitates porting the code to a distributed-memory parallel computer. Earlier work on Navier-Stokes integrators has suggested that non-staggered mesh codes can be numerically unstable because of pressure oscillations [17,20]. Our results below show that an algorithm to integrate the Boussinesq equations on a non-staggered mesh can be numerically stable.

The use of a single non-staggered mesh also helps to explain the efficiency of the algorithm. Using a standard operator splitting and projection method together with secondorder-accurate finite differences $[21,22]$ on a uniform three-dimensional mesh, the advancement of the velocity, temperature, and pressure fields at each time step requires the numerical solution of four Helmholtz equations and one Poisson equation. Because these elliptic equations and their boundary conditions are separable, they can be solved efficiently using fast direct methods from the FISHPACK library [23, 24], with a complexity per problem of $O(N \log (N))$, where $N$ is the total number of mesh points. Fast direct methods are more efficient than most iterative methods on a single processor [25], and have the additional advantage that no internal parameters need to be adjusted to obtain convergence. However, fast direct methods are not applicable to complex geometries, to problems with spatially varying parameters, or to complicated boundary conditions that lead to nonseparable equations.

The remainder of this paper is organized as follows. In Section II, we discuss details of our algorithm, namely how the fields and equations are discretized and how the resulting equations are solved. In Section III, we discuss the convergence properties of the algorithm and its efficiency for several representative two- and three-dimensional convection problems. We confirm empirically the second-order accuracy of the solution and examine how the largest time step allowed by stability varies with Prandtl number and with Rayleigh number. Finally, Section IV presents our conclusions and suggests some avenues for further algorithmic improvements. Applications of the algorithm to study quasiperiodic dynamics and spiral defect chaos in three-dimensional boxes can be found in Refs. [4,5]. 


\section{DETAILS OF THE ALGORITHM}

\section{A. Equations and Boundary Conditions}

Our goal is to integrate the Boussinesq equations that describe incompressible buoyancydriven Rayleigh-Bénard convection with an external force $\mathbf{f}$. These equations can be written in the dimensionless form [26]

$$
\begin{aligned}
\partial_{t} T(t, x, y, z) & =[-(\mathbf{v} \cdot \nabla) T]+\nabla^{2} T, \\
\partial_{t} \mathbf{v}(t, x, y, z) & =[-(\mathbf{v} \cdot \nabla) \mathbf{v}+\sigma R T \hat{\mathbf{z}}+\sigma \mathbf{f}]+\sigma \nabla^{2} \mathbf{v}-\nabla p, \\
\nabla \cdot \mathbf{v} & =0 .
\end{aligned}
$$

The variables $x$ and $y$ denote the horizontal coordinates, while the $z$ variable denotes the vertical coordinate, with the unit vector $\hat{\mathbf{z}}$ pointing in the direction opposite to the gravitational acceleration. The field $\mathbf{v}=\left(v_{x}, v_{y}, v_{z}\right)$ is the velocity field at point $(x, y, z)$ at time $t$, while $p$ and $T$ are the pressure and temperature fields respectively. The dimensionless parameters $\sigma$ and $R$ denote the Prandtl and Rayleigh numbers respectively. The vector field $\mathbf{f}(t, \mathbf{x}, T, \mathbf{v})$ is some external force, e.g., a Coriolis force $\mathbf{f}=2 \mathbf{v} \times \boldsymbol{\Omega}$ arising from a rigid rotation of the convection cell with constant angular velocity $\Omega=\Omega \hat{\mathbf{z}}$. The terms grouped in brackets in Eqs. (1) and (2) are those containing nonlinear terms or linear terms with low-order spatial derivatives, and will be integrated explicitly by the operator splitting method described below.

We would like to integrate Eqs. (1)-(3) in a box geometry defined by the region

$$
-\frac{\Gamma_{x}}{2} \leq x \leq \frac{\Gamma_{x}}{2}, \quad-\frac{\Gamma_{y}}{2} \leq y \leq \frac{\Gamma_{y}}{2}, \quad-\frac{1}{2} \leq z \leq \frac{1}{2},
$$

where $\Gamma_{x}$ and $\Gamma_{y}$ are the aspect ratios in the $x$ and $y$ directions respectively (the depth of the fluid has length 1). A no-slip velocity condition on all material walls is assumed

$$
\mathbf{v}=0,
$$

and the temperature $T$ is constant on the bottom and top plates, 


$$
T= \pm \frac{1}{2} \quad \text { for } \quad z=\mp \frac{1}{2}
$$

The code allows the temperature on any opposing pair of lateral walls to be periodic, or to satisfy on each lateral wall an arbitrary Dirichlet boundary condition (e.g., a thermally conducting wall corresponding to a linear conducting profile of the form $T=a+b z$, where $a$ and $b$ are constants), or an arbitrary Neumann condition (e.g., a thermally insulating wall with $\partial_{n} T=0$, where $\partial_{n}$ is the normal derivative to the boundary at a given point). To simplify the following discussion, we will consider only the case of insulating sidewalls

$$
\partial_{n} T=0, \quad \text { on lateral walls, }
$$

since the other cases involve just simple modifications. Although the pressure field $p$ formally has no associated boundary condition since it does not satisfy a dynamic equation, we will be imposing a Neumann boundary condition on $p$ as we explain below (see Eq. (17)).

\section{B. The Time Integration Method}

We next discuss the time integration method, since its structure can be explained before having to specify a spatial representation for the fields. In the following subsection, we discuss how the fields and equations are discretized and the latter solved using secondorder-accurate finite differences on a uniform spatial mesh.

Our time integration method uses a standard operator splitting and projection method $[21,22]$, in which the nonlinear terms containing lower-order or no spatial derivatives are integrated explicitly, then the linear diffusion operators are integrated implicitly, and finally the pressure term $-\nabla p$ is integrated to project the velocity field at the next time step into the space of divergence-free velocity fields. Operator splitting has two benefits. First, the evolution equations for $T$ and for the velocity components $v_{i}$ are decoupled from one another, which simplifies the overall algorithm and substantially reduces the total computer

memory needed. Second, operator splitting allows larger time steps since the largest time 
step $\Delta t$ allowed by stability is bounded by a first power of the spatial resolution $\Delta x$, rather than by a second power as would be the case for a fully explicit method.

Let us assume that, at the $n$th time step $t_{n}=n \Delta t$ with $n \geq 0$, initial fields $T^{n}$ and $\mathbf{v}^{n}$ are known that are consistent with the boundary conditions Eqs. (5)-(7). These fields are then advanced to the future values $T^{n+1}$ and $\mathbf{v}^{n+1}$ at time $t_{n+1}=t_{n}+\Delta t$ as follows:

1. The nonlinear advective term $N_{T}[T, \mathbf{v}]=-(\mathbf{v} \bullet \nabla) T$ of Eq. (1) is integrated explicitly using a second-order-accurate Adams-Bashforth method

$$
T^{*}=T^{n}+\frac{\Delta t}{2}\left(3 N_{T}\left[T^{n}, \mathbf{v}^{n}\right]-N_{T}\left[T^{n-1}, \mathbf{v}^{n-1}\right]\right), \quad n \geq 0
$$

to produce an intermediate field $T^{*}$. Here $T^{n-1}$ and $\mathbf{v}^{n-1}$ denote field values stored from the previous time step $t_{n-1}=t_{n}-\Delta t$. For the first time step $n=0$ only, a secondorder-accurate single-step integrator, Heun's method [27], is used in place of AdamsBashforth to avoid the dependence on the unavailable field values at time $t=-\Delta t$.

2. The intermediate field $T^{*}$ is then advanced to the temperature field $T^{n+1}$ at time $t_{n+1}$ by using $T^{n}$ as initial data for an implicit Crank-Nicolson step applied to the diffusion term in Eq. (1):

$$
\frac{T^{n+1}-T^{*}}{\Delta t}=\frac{1}{2}\left(\nabla^{2} T^{n+1}+\nabla^{2} T^{n}\right)
$$

This can be written as a constant-coefficient Helmholtz equation for the future field $T^{n+1}$ :

$$
\left(1-\frac{\Delta t}{2} \nabla^{2}\right) T^{n+1}=T^{*}+\frac{\Delta t}{2} \nabla^{2} T^{n}
$$

and is solved with the boundary conditions Eqs. (6) and (7) applied to $T^{n+1}$.

3. Three similar pairs of explicit and implicit steps are then executed successively, first for the velocity component $v_{x}$, then for $v_{y}$, and then for $v_{z}$. The explicit steps advance the velocity field $\mathbf{v}^{n}$ to an intermediate field $\mathbf{v}^{*}$, and then the implicit steps advance $\mathbf{v}^{*}$ 
to a second intermediate field $\mathbf{v}^{* *}$. If we denote by $N_{i}[T, \mathbf{v}]$ the expressions in brackets of Eq. (2) for $i=x, y$, and $z$, then each explicit step has the form

$$
v_{i}^{*}=v_{i}^{n}+\frac{\Delta t}{2}\left(3 N_{i}\left[T, \mathbf{v}^{n}\right]-N_{i}\left[T, \mathbf{v}^{n-1}\right]\right) .
$$

Heun's single-step method is again used at time $t_{0}=0$ to avoid unavailable field values at time $t_{-1}=-\Delta t$. Each field $v_{i}^{n}$ is next used as initial data for an implicit CrankNicolson step that yields a constant-coefficient Helmholtz equation for the field $v_{i}^{* *}$ :

$$
\left(1-\frac{\sigma \Delta t}{2} \nabla^{2}\right) v_{i}^{* *}=v_{i}^{*}+\frac{\sigma \Delta t}{2} \nabla^{2} v_{i}^{n} .
$$

This is solved with the no-slip boundary condition $v_{i}^{* *}=0$ on all surfaces, Eq. (5).

4. An incompressible velocity field $\mathbf{v}^{n+1}$ at time $t_{n+1}$ is obtained from the field $\mathbf{v}^{* *}$ by integrating the final operator step

$$
\partial_{t} \mathbf{v}=-\nabla p,
$$

with initial data $\mathbf{v}^{* *}$, followed by a projection method $[22,28]$. We approximate the time derivative in Eq. (13) with a first-order-accurate stencil,

$$
\frac{\mathbf{v}^{n+1}-\mathbf{v}^{* *}}{\Delta t}=-\frac{1}{2}\left(\nabla p^{n+1}+\nabla p^{n}\right),
$$

apply the divergence operator to both sides, and then use Eq. (3) in the form $\nabla \cdot \mathbf{v}^{n+1}=0$. This yields a Poisson equation for the pressure field $p$ :

$$
\nabla^{2} p^{n+1}=-\nabla^{2} p^{n}+\frac{2}{\Delta t} \nabla \cdot \mathbf{v}^{* *} .
$$

Once $p$ is known by solving Eq. (15), we obtain $\mathbf{v}^{n+1}$ from Eq. (14) in the form

$$
\mathbf{v}^{n+1}=\mathbf{v}^{* *}-\frac{\Delta t}{2}\left(\nabla p^{n+1}+\nabla p^{n}\right) .
$$

Although mathematically there is no boundary condition for $p$-and by discretizing first space and then time, a boundary condition for $p$ can be avoided as explained in Refs. $[21,29,30]$ —we will solve Eq. (15) with the Neumann condition 


$$
\partial_{n} p^{n+1}=0 \quad \text { on all walls, }
$$

since this allows us to use a fast direct method to solve Eq. (15). There is a substantial literature concerning the appropriateness and accuracy of the boundary condition Eq. (17) $[31,32]$. Rather than review this literature, we simply point out that a Neumann pressure boundary condition has been shown by previous researchers to produce acceptably accurate results for problems in which the fluid is confined by no-slip surfaces, and we show directly in Section III that our algorithm is second-order accurate in space and second-order accurate in time for several representative problems.

The most time consuming part of this algorithm is, by far, solving the four Helmholtz equations Eq. (10) and Eq. (12) (for $i=x, y$, and $z$ ) and solving the Poisson equation Eq. (15).

\section{Discretization on a Uniform Mesh}

The explicit and implicit steps of the previous section-Eqs. (8) and (10), Eqs. (11) and (12), and Eqs. (15) and (16) - are carried out by discretizing the fields and equations on a single non-staggered mesh of points

$$
\mathbf{x}_{i j k}=(i \Delta x, j \Delta y, k \Delta z)
$$

that is commensurate with the sides of the box Eq. (4). The mesh indices $(i, j, k)$ satisfy

$$
-\frac{N_{x}}{2} \leq i \leq \frac{N_{x}}{2}, \quad-\frac{N_{y}}{2} \leq j \leq \frac{N_{y}}{2}, \quad-\frac{N_{z}}{2} \leq k \leq \frac{N_{z}}{2}
$$

The aspect ratios $\left(\Gamma_{x}, \Gamma_{y}\right)$ and the positive integers $\left(N_{x}, N_{y}, N_{z}\right)$ are specified as input to the

code, and the corresponding spatial resolutions $(\Delta x, \Delta y, \Delta z)$ are then determined from the relations $\Delta x=\Gamma_{x} / N_{x}, \Delta y=\Gamma_{y} / N_{y}$, and $\Delta z=1 /\left(N_{z}\right)$. For large-aspect-ratio convection problems, typically $\Delta x=\Delta y>\Delta z$ since the $x$ and $y$ directions are equivalent and there is a finer structure in the vertical direction caused by the close opposing horizontal plates. 
At all mesh points Eq. (18) interior to the box, the first- and second-order spatial derivatives are approximated using centered second-order-accurate 3-point finite-difference stencils. If $u_{i j k}=u\left(\mathbf{x}_{i j k}\right)$ denotes the values of a field $u(\mathbf{x})$ at the mesh points, then the partial derivative $\partial_{x} u$ at $\mathbf{x}_{i j k}$ is approximated by

$$
\left[\partial_{x} u\right]_{i j k} \approx \frac{u_{(i+1) j k}-u_{(i-1) j k}}{2 \Delta x}
$$

with similar expressions for $\partial_{y} u$ and $\partial_{z} u$. The Laplacian of $u$ at $\mathbf{x}_{i j k}$ is approximated by the usual 7-point stencil

$$
\begin{gathered}
{\left[\nabla^{2} u\right]_{i j k} \approx \frac{u_{(i+1) j k}-2 u_{i j k}+u_{(i-1) j k}}{\Delta x^{2}}+\frac{u_{i(j+1) k}-2 u_{i j k}+u_{i(j-1) k}}{\Delta y^{2}}} \\
+\frac{u_{i j(k+1)}-2 u_{i j k}+u_{i j(k-1)}}{\Delta z^{2}} .
\end{gathered}
$$

Nonsymmetric finite-differences are needed to evaluate expressions on those boundaries for which a Neumann condition holds (we will call these "Neumann boundaries") since field values outside the domain are not available. Thus the right side of the Helmholtz equation, Eq. (10), needs to be evaluated on the Neumann boundaries for which Eq. (7) holds. The value of $\nabla^{2} T^{*}$ can be approximated there to second-order accuracy by using one-sided 4point finite-difference approximations for the 2nd-order derivatives, e.g.,

$$
\left[\partial_{x}^{2} T^{*}\right]_{0 j k} \approx \frac{2 T_{0 j k}^{*}-5 T_{1 j k}^{*}+4 T_{2 j k}^{*}-T_{3 j k}^{*}}{\Delta x^{2}}
$$

with similar expressions for $\partial_{x}^{2} T^{*}$ at $x=\Gamma_{x}$, and for $\partial_{y}^{2} T^{*}$ on the $y=0$ and $y=\Gamma_{y}$ boundaries. The divergence $\nabla \cdot \mathbf{v}^{* *}$ on the right side of the pressure equation, Eq. (15), can be approximated to second-order accuracy using the Dirichlet data Eq. (5) and interior field values by replacing Eq. (20) with the following 3-point one-sided finite difference

$$
\left[\partial_{x} v_{x}\right]_{0 j k} \approx \frac{-3\left(v_{x}\right)_{0 j k}+4\left(v_{x}\right)_{1 j k}-\left(v_{x}\right)_{2 j k}}{2 \Delta x}=\frac{4\left(v_{x}\right)_{1 j k}-\left(v_{x}\right)_{2 j k}}{2 \Delta x}
$$

with similar expressions for $\partial_{y} v_{y}$ and $\partial_{z} v_{z}$. The advective derivative $-(\mathbf{v} \bullet \nabla) T$ in Eq. (8) vanishes on these Neumann walls since $\mathbf{v}$ does, and so the explicit steps do not require special treatment. 
Given the discretizations Eqs. (20)-(24), the explicit time steps Eqs. (8) and (11) are easily evaluated at all interior points and on the Neumann boundaries. For the implicit steps, the right sides of Eqs. (10) and (12) are also evaluated on the interior mesh points and on the Neumann boundaries. These right sides are then used as input to the FISHPACK [24] fast direct solvers hw3crt in three dimensions or hwscrt in two dimensions. Also provided as input to the FISHPACK solvers are the corresponding boundary conditions, Eqs. (6) and (7) for T, Eq. (5) for the velocity components, and Eq. (17) for $p$. The FISHPACK solvers return second-order-accurate values (with respect to the spatial resolution) of $T, \mathbf{v}$, and $p$ on the mesh $\mathbf{x}_{i j k}$.

We conclude this section with the observation that the discrete velocity field $\mathbf{v}^{n+1}$ obtained from the concluding step Eq. (16) is only approximately divergence-free even on the mesh points $\mathbf{x}_{i j k}$, i.e., $\nabla \cdot \mathbf{v}^{n+1}=O\left(h^{2}\right)$ where $h$ is the larger of the spatial resolutions $\Delta x$, $\Delta y$, and $\Delta z$. This is because the discrete approximation Eq. (20) for the pressure gradient in Eq. (16) is not consistent with the discretization Eq. (21) used to approximate the Laplacian $\nabla \cdot \nabla$ on the left side of Eq. (15). The discrete Laplacian can be considered as arising from the evaluation of pressure gradients from pairs of nearest neighbor points as follows

$$
\begin{aligned}
{\left[\partial_{x}^{2} p\right]_{i j k} } & =\frac{1}{\Delta x}\left(\left[\partial_{x} p\right]_{(i+1 / 2) j k}-\left[\partial_{x} p\right]_{(i-1 / 2) j k}\right) \\
& =\frac{1}{\Delta x}\left(\frac{p_{(i+1) j k}-p_{i j k}}{\Delta x}-\frac{p_{i j k}-p_{(i-1) j k}}{\Delta x}\right) .
\end{aligned}
$$

In contrast, Eq. (16) evaluates the pressure gradient at a point using a finite difference Eq. (20) that spans three mesh points.

\section{ACCURACY AND EFFICIENCY OF THE ALGORITHM}

In this section, we discuss several tests that quantify the accuracy of the above algorithm for a convecting fluid in a two-dimensional rectangular domain with periodic sidewalls and in a three-dimensional rectangular domain with perfectly insulating sidewalls, Eq. (7). We first confirm the second-order accuracy of the code with respect to the spatial and time 
resolutions by studying how the temperature and velocity fields converge with increasing spatial and time resolutions respectively. We next show empirically how the maximum stable time step varies with the Rayleigh number $R$ and the Prandtl numbers $\sigma$. We then calculate the critical Rayleigh number $R_{c}$ and plot the Nusselt number $N(R)$ as a function

of the Rayleigh number R, and obtain good agreement with an analytical expression [34] and with a spectral code [35]. Finally, we show the spatial structure of the fields near onset, to allow comparison with experiment [36] and with other codes.

We note that, on a workstation with a $667 \mathrm{MHz} 21264 \mathrm{~A}$ 64-bit Alpha processor, a square box with aspect ratio $\Gamma=40$ and spatial resolution $\Delta x=\Delta y=\Delta z=1 / 8$ takes about $4.8 \mathrm{~s}$ per time step of $\Delta t=0.001 t_{v}$. This corresponds to 80 minutes per vertical diffusion time $t_{v}$ and 90 days per horizontal diffusion time $t_{h}$, so this code is too slow to explore $\Gamma>20$ cells over time scales exceeding a horizontal diffusion time. We discuss two ways of improving the efficiency of the code in our concluding comments of Section IV.

\section{A. Second-Order Convergence With Respect to the Spatial and Time Resolutions}

We begin by showing that the order of convergence $p$ of the code is asymptotically 2 (second order) in the limits of sufficiently fine spatial and time resolutions. By definition, the convergence with respect to spatial resolution is of order $p$ if $\left\|u_{h}-u_{\text {exact }}\right\|=O\left(h^{p}\right)$ in

the limit $h \rightarrow 0$, where $\|u\|=\sqrt{\sum_{i j} u_{i j}^{2}}$ denotes the Euclidean norm of a field $u$ on the spatial mesh, $h=\Delta x=\Delta z$ is the uniform spatial resolution in the $x$ and $z$ directions of a two-dimensional box, $u_{h}(x, z)$ denotes a discrete numerical field on a mesh of resolution $h$, and $u_{\text {exact }}(x, z)$ is the unknown exact field on the spatial mesh. By writing $u_{h}(x, z)=$ $u_{\text {exact }}(x, z)+C(x, z) h^{p}$ in the limit $h \rightarrow 0$, for some function $C$ independent of $h$, we deduce that the order $p$ can be estimated by examining the quantity

$$
p_{h}=\log _{2}\left(\frac{\left\|u_{4 h}-u_{2 h}\right\|}{\left\|u_{2 h}-u_{h}\right\|}\right),
$$

in the limit $h \rightarrow 0$. The estimate Eq. (27) involves field values at the three levels of resolution $4 h, 2 h$, and $h$, coarsest to finest. A similar definition for the order of convergence 
with respect to time resolution can be made if the spatial mesh $h$ is replaced by the time step $g \equiv \Delta t$

$$
p_{g}=\log _{2}\left(\frac{\left\|u_{4 g}-u_{2 g}\right\|}{\left\|u_{2 g}-u_{g}\right\|}\right)
$$

We first studied the convergence with respect to the spatial mesh $h$ for a two-dimensional box with periodic sidewalls, for parameter values $\Gamma_{x}=2 \pi / q_{c}=2.016, R=1725.0 \approx 1.01 R_{c}$, and $\sigma=0.71$. The initial conditions consisted of a small random perturbation about the linearly conducting state $T_{0}=-z, \mathbf{v}_{0}=\left(u_{0}, w_{0}\right)=\mathbf{0}$, and these were integrated until a stationary state was attained consisting of two rolls at the critical wave number $q_{c}$. For this small cell, an integration time of $8 t_{v}$ was sufficient for the dynamics to become stationary. We then studied the temperature field, $T_{h}(x, z)$, and the $x$-component of the velocity field, $u_{h}(x, z)$, for different spatial resolutions $N=\Gamma_{x} / h=16,32,64$, and 128. The time step $\Delta t$ was set respectively to the values $\Delta t=0.01,0.005,0.0025$, and 0.00125 since the operating splitting makes the largest stable time step proportional to $h$. Table I summarizes the values of the limit Eq. (27) and shows that indeed $p_{h} \rightarrow 2$ as $h \rightarrow 0$, i.e., the code is asymptotically second-order accurate with respect to the spatial resolution $h$.

We have also studied the convergence with respect to the time step $g$ for a threedimensional box with perfectly insulating sidewalls and for parameter values $\Gamma_{x}=\Gamma_{y}=2$, $R=1725.0 \approx 1.01 R_{c}$, and $\sigma=0.71$. The initial condition consisted of small random thermal perturbations, and these were integrated up to 20 diffusion times at which point the state became stationary. For various time resolutions $g=\Delta t=0.0001,0.00005$, and 0.000025, all with a space resolution of $N=64$, the convergence was found (using Eq. (28)) to be $p=1.68$. This provides evidence that the code is indeed asymptotically second-order accurate with respect to the time resolution $g$.

\section{B. Dependence of Maximum Stable Time Step on Rayleigh and Prandtl Numbers}

Since an important practical feature of any production code is the largest time step that can be taken before numerical instability occurs, we have studied the maximum stable 
time step as a function of the Rayleigh and Prandtl numbers. A three-dimensional box with periodic sidewalls and aspect ratio $\Gamma_{x}=\Gamma_{y}=2$ was used, with a spatial resolution $\Delta x=16$. The Euclidean norm of the temperature field, $\|T\|$, was calculated for various values of $\Delta t$ each time after a interval of 20 vertical diffusion times so that transients decayed. The maximum stable time step was then defined as the value of $\Delta t$ such that $\|T\|$ remains bounded, i.e., $\|T\|<10^{5}$.

In Fig. 1(a), we plot the maximum stable time step as a function of Rayleigh number for the two Prandtl number values $\sigma=1$ (square symbols) and $\sigma=10$ (cross symbols). We see that the maximum stable time step decreases rapidly with increasing Rayleigh number. This is to be expected since the magnitude of the velocity and temperature fields increase with increasing $R$. In fact, a best log-log fit to the data yields the relation

$$
\max (\Delta t) \propto R^{\alpha}
$$

where $\alpha=-1.2$ when $\sigma=1$, and $\alpha=-1.3$ when $\sigma=10$.

In Fig. 1(b), we plot the maximum stable time step as a function of Prandtl number for fixed Rayleigh numbers $R=2048$ (square symbols) and $R=8192$ (cross symbols). For $R=2048$, the maximum stable time step decreases toward both small and large Prandtl numbers. For $R=8192$, the maximum stable time step decreases toward small Prandtl numbers but is approximately constant at large Prandtl numbers. The smaller time step needed at small Prandtl numbers can be attributed to the more dynamical nature of the convective flow at small Prandtl numbers, such as the presence of spiral defect chaos [33].

\section{Estimate of the Critical Rayleigh Number $R_{c}$}

A linear stability analysis of the Boussinesq equations about the linearly conducting profile between two infinite horizontal no-slip plates shows that the critical Rayleigh number $R_{c} \approx 1707.76$ with critical wave number $q_{c} \approx 3.117$, and that the values of $R_{c}$ and $q_{c}$ are

independent of the Prandtl number $\sigma[2]$. We tested these predictions and so validated the 
code by using a two-dimensional box of aspect ratio $\Gamma_{x}=2 \pi / q_{c}=2.016$, with periodic sidewalls, for Prandtl number $\sigma=0.71$. We used a uniform spatial resolution $h=1 / N=$ $\Delta x=\Delta z$ and varied the number $N$ of mesh points.

The critical Rayleigh number $R_{c}$ was estimated as the approximate value of $\mathrm{R}$ for which the growth rate $\lambda=\lambda(R)$ of a small-amplitude (0.01) random perturbation about the linear profile interpolated to zero as a function of $\mathrm{R}$. Thus for a sufficiently tiny initial perturbation of the conducting profile, there is a time interval over which the z-velocity component $w$ grows approximately exponentially

$$
\|w(t, x, z)\| \approx c(R) e^{\lambda t}
$$

where $\lambda$ is the growth rate, and $c$ is independent of $t$ but can vary with $\mathrm{R}$. For $R>R_{c}$, the growth rate is positive, for $R<R_{c}$, the growth rate is negative and interpolating between known positive and negative values provides an estimate of $R_{c}$, for which $\lambda=0$.

Our protocol was to set $R=R_{+}=1725=1.01 R_{c}$ just above onset, set the initial velocity field to zero, $\mathbf{v}_{0}=\left(u_{0}, w_{0}\right)=\mathbf{0}$, and set the initial temperature field $T_{0}=-z+\delta T$ to a tiny random perturbation $\delta T(x, z)$ of the linear profile $T=-z$, with $|\delta T| \leq 0.01$. The initial conditions were then integrated for a short time and the growth rate estimated from the formula

$$
\lambda_{+} \approx \frac{\ln \left(\left\|w\left(t_{2}, x, z\right)\right\| /\left\|w\left(t_{1}, x, z\right)\right\|\right)}{t_{2}-t_{1}}
$$

where $t_{2}$ and $t_{1}<t_{2}$ are two times during the exponential growth of the magnitude of the $z$-component of the velocity field $w$. The calculation was then repeated with the same initial condition but for $R=R_{-}=1691=0.99 R_{c}$ to estimate a decay rate $\lambda_{-}$. The critical Rayleigh number was then estimated as the zero of the line joining the points $\left(R_{+}, \lambda_{+}\right)$ and $\left(R_{-}, \lambda_{-}\right)$. The estimated critical Rayleigh numbers $R_{c}$ as a function of the number of mesh points $N$ are summarized in Table II. The values are correct to a relative error of better than one percent for the finest spatial resolution, confirming the correctness and convergence of the discretization and of the solution technique. 


\section{The Nusselt number versus Rayleigh number Curve $N(R)$}

Another way to characterize the accuracy of a convection code is by the dimensionless Nusselt number $N(t, R, \sigma)$, which is the instantaneous global vertical heat transport through the fluid layer, normalized to the heat transport arising from thermal conduction alone. For the dimensionless variables used in Eqs. (1)-(3) above, the Nusselt number can be expressed in the form $[26]$

$$
N=1+\left\langle w\left(T-T_{\text {cond }}\right)\right\rangle
$$

where $w$ is the $z$-component of the velocity field and $T_{\text {cond }}=-z$ is the temperature profile

of the linear conducting state with $\mathbf{v}=\mathbf{0}$. The brackets $\langle\cdots\rangle$ denote an average of a quantity over the horizontal coordinates. Sufficiently close to onset, numerical values of $N$ can be compared with an analytical expression [34] that is valid asymptotically in the limit $R-R_{c} \rightarrow 0^{+}$.

We have evaluated Eq. (32) for the two-dimensional domain with periodic sidewalls of the previous section, with parameters $\Gamma_{x}=2.016, \sigma=0.71$, and $N=16$. Starting from a small perturbation of the linear profile, we integrated until a stationary state was attained, and then evaluated Eq. (32) for the stationary state. Fig. 2 shows how $N$ empirically

varies with $\mathrm{R}$, and we compare this curve with the analytical result of Schlüter et al [34], and with numerical values obtained by Clever and Busse[35], who used a two-dimensional spectral code with periodic side walls. The agreement is good in both cases and confirms the correctness and accuracy of the code.

\section{E. Spatial Structure of the Numerical Solutions}

We conclude this section with a few examples of the spatial structure of the fields obtained from the code, to show that the fields are physically reasonable when adequately resolved, and are qualitatively in agreement with other codes and with experiment [36]. 
for $\sigma=0.71$, Fig. 3 shows contours of constant temperature $T$ and for two values of the Rayleigh number, $R=2500$ in (a) and $R=10^{4}$ in (b). Warm fluid ascends in the middle of the cell and descends as cooler fluid on both sides. As $R$ increases, a thermal boundary layer forms at the top and bottom plates, creating a finer spatial structure that will require eventually a decrease in the vertical spatial mesh size $\Delta z$. Fig. 4(a) shows the corresponding velocity field $\mathbf{v}=(u, w)$, while Fig. 4(b) shows the vertical component $w$ through the midline of the cell. The occurrence of two square-shaped convection cells of opposite vorticity is in good agreement with experiment [36].

Fig. 5 shows constant temperature contours in a three-dimensional box with insulating sidewalls at time $t=200 t_{v}$, for parameters $\Gamma=16, R=2500, \sigma=0.71$, and $h=\Delta x=$ $\Delta y=\Delta z=1 / 8, \Delta t=0.01$. In agreement with experiment [38] and with calculations on the Swift-Hohenberg model of convection [39], the rolls are approximately normal to the lateral walls and the pattern consists of two diagonally opposite foci. For slightly higher $R=$ 8500, Fig. 5(b) shows that the oscillatory instability commenced in the form of ripples that propagate along the length of the rolls. The occurrence of the oscillatory instability and its spatial form are in good agreement with the linear stability analysis of Busse and collaborators [26] and with experiment.

\section{CONCLUSIONS}

We have described and characterized a semi-implicit finite-difference algorithm for integrating the Boussinesq equations in two- and three-dimensional boxes, with sidewalls that are periodic, thermally insulated, or thermally conducting. Our approach is useful for simple geometries like a box, cylinder, torus, and annulus, with boundary conditions such that various linear operators are separable so that fast direct methods can be applied. The resulting algorithm is sufficiently efficient that aspect ratios up to $\Gamma \approx 20$ can be studied on a single-processor workstation over several days. We verified that the code was second-orderaccurate with respect to the spatial and time resolutions, and that it gave good agreement 
for the critical Rayleigh number and for the Nusselt number versus Rayleigh number curve near onset.

The most significant feature of our algorithm is the use of a single non-staggered mesh for discretizing the equations and fields (velocity, temperature, and pressure). The use of a single mesh simplifies the writing and validation of the code, and facilitates adding new physical terms like a Coriolis force. The single mesh also allowed the use of fast direct methods from the FISHPACK library [24] to solve the Helmholtz and Poisson equations associated with the implicit part of each time step. We found that numerical integrations of the Boussinesq equations were stable on a single mesh despite results of some previous papers that suggested that a non-staggered Navier-Stokes code could be unstable because of pressure oscillations,

Although the algorithm is useful and has been successfully applied to several problems [4, $5]$, there are two ways that the algorithm could be improved for the future study of largeaspect-ratio Rayleigh-Bénard convection. First is to parallelize the code for a distributed memory parallel computer so that aspect ratios comparable to the largest experiments $(50<$ $\Gamma<100)$ could be studied. This is technically straightforward and would involve, first, distributing the arrays that represent the fields over the various processors, and, second, replacing the fast direct solvers with iterative methods for sparse matrices. Because of the simpler data structures and reduced communication overhead associated with a finitedifference discretization, the parallelized algorithm will likely be more efficient for simple geometries and for simple boundary conditions than the parallel spectral element method of Refs. [13, 14].

Because time integration algorithms involve sequential steps, parallelizing a code allows a larger spatial domain, but not a longer observation time, to be studied for a fixed amount of wall-clock time. A second helpful improvement would be to increase the efficiency of the time integration method close to the onset of convection so that larger time steps can be taken for a given computational effort. A weakness of the operating splitting used in most convection codes - finite difference, spectral, and spectral-element - is that the explicit 
integration of the advection terms imposes a bound on the time step of the form $C \epsilon^{-1 / 2} \Delta x$ where $C$ is a constant and $\epsilon=\left(R-R_{c}\right) / R_{c}$ is the reduced Rayleigh number. This bound is independent of the spatial resolution and diverges less rapidly in the limit $\epsilon \rightarrow 0^{+}$than the physical time scale, which is proportional to $\epsilon^{-1}$. It would be interesting to explore whether a more sophisticated explicit time-stepping technique such as a matrix exponential method $[40,41]$ or a fully implicit method $[42,43]$ may succeed in allowing larger time steps that are commensurate with the physical time scale while retaining the efficiency of the present code.

\section{ACKNOWLEDGMENTS}

We would like to thank Michael Cross, Paul Fischer, and Mark Paul for helpful discussions, and the Department of Energy for supporting this research under grant DE-FT0298ER14892. 


\section{FIGURES}

FIG. 1. (a) Plot of the maximum stable time step as a function of Rayleigh number. The Prandtl number is kept constant at $\sigma=1$ (square symbols) and $\sigma=10$ (for cross symbols). The cell has aspect ratio $\Gamma_{x}=\Gamma_{y}=2$ and periodic sidewalls. The mesh resolution is $\Delta x=1 / 16$. Small random perturbations in the temperature field are used as initial conditions. The simulation is run until 20 vertical diffusion times, at which time the Euclidean norm $\|T\|$ is then calculated. The value of $\Delta t$ such that this norm becomes greater than $10^{5}$ is defined as the maximum stable time step. (b) Plot of the maximum stable time step as a function of Prandtl number. The Rayleigh number was kept constant at $R=2048$ (square symbols) and $R=8192$ (cross symbols). The same aspect ratio, mesh resolution, and initial conditions as in (a) were used.

FIG. 2. (a) Comparison of numerical (square symbols) and theoretical (cross symbols) Nusselt numbers versus Rayleigh number. The numerical values come from time-independent two-dimensional nonlinear states (at time $t=12$ ) with periodic sidewalls. The parameters have the values $\Gamma_{x}=2, R=2500, \sigma=0.71, \triangle x=\triangle z=1 / 16(N=16)$, and $\triangle t=0.01$. The initial state was a small random perturbation of the linear conducting profile. The theoretical values come from an asymptotic expansion [34]. (b) Comparison of Nusselt number versus Rayleigh number obtained numerically for our algorithm (squares) and for a spectral code of Clever and Busse [35] $(*)$. The agreement is better than $3 \%$.

FIG. 3. (a) Contour lines of the temperature field $T(x, z)$ observed at time $t=12$ in a two-dimensional box of aspect ratio $\Gamma_{x}=2$ with periodic sidewalls. The parameters have values $R=2500, \sigma=0.71, \triangle x=\triangle z=1 / 16(N=16)$, and $\triangle t=0.01$. (b) Contour lines of the temperature field observed in a simulation using the same geometry and spatial resolution as in (a) but for $R=10^{4}$ and time step $\triangle t=0.0025$. 
FIG. 4. (a) Time-independent velocity field $\mathbf{v}(x, z)$ at time $t=12$ for $R=2500$, for the same geometry and resolution as Fig. 3. The steady state consists of two convection rolls at the critical wavenumber $q_{c}=3.117$. (b) Vertical velocity component $w(x, z=0)$ through the midline of the cell, indicating the range of $w$.

FIG. 5. (a) Weakly time-dependent temperature contours at the midplane, $T(x, y, z=0)$, at time $t=200$ obtained from a three-dimensional box of aspect ratio $\Gamma_{x}=\Gamma_{y}=16$ with no-slip and insulating boundary conditions, Eqs. (5) and (7). Parameter values are $R=2500, \sigma=0.71$, $\Delta x=\Delta y=\Delta z=1 / 8$, and $\Delta t=0.01$. (b): Time-dependent temperature contours at the midplane, $T(x, y, z=0)$, for the same geometry and resolutions as in (a) but for $R=8500$ for which the rolls are unstable to the oscillatory instability, which shows up as propagating ripples along the rolls. The time-averaged Nusselt number $\langle N\rangle=2.27$ is larger than for (a), for which the value is $\langle N\rangle=1.44$. 


\section{REFERENCES}

*E-mail correspondence should be sent to hsg@phy.duke.edu.

$\dagger$ Also Duke University's Center for Nonlinear and Complex Systems.

[1] E. Bodenschatz, W. Pesch, and G. Ahlers, Ann. Rev. Fluid Mech. 32, 709 (2000).

[2] M. C. Cross and P. C. Hohenberg, Rev. Mod. Phys. 65, 851 (1993).

[3] M. C. Cross and P. C. Hohenberg, Science 263, 1569 (1994).

[4] M.-C. Lai, K.-H. Chiam, M. C. Cross, and H. S. Greenside, in 18th Symposium on Energy Engineering Sciences, Department of Energy (Argonne National Laboratory, Argonne National Laboratory, 2000), preprint http://arXiv.org/ps/nlin.PS/0005058.

[5] K.-H. Chiam, M. C. Cross, and H. S. Greenside, Phys. Rev. E (2003), to appear.

[6] D. A. Egolf, I. V. Melnikov, W. Pesch, and R. E. Ecke, Nature 404, 733 (2000).

[7] W. Decker, W. Pesch, and A. Weber, Phys. Rev. Lett. 73, 648 (1994).

[8] W. Pesch, Chaos 6, 348 (1996).

[9] W. Arter and A. Newell, Physics of Fluids 31, 2474 (1988).

[10] H. Tomita and K. Abe, Physics of Fluids 11, 743 (1999).

[11] H. Xi, X.-J. Li, and J. D. Gunton, Phys. Rev. Lett. 78, 1046 (1997).

[12] M. R. Paul, M. C. Cross, P. F. Fischer, and H. S. Greenside, Phys. Rev. Lett. 87, 154501 (2001).

[13] M. R. Paul, M. C. Cross, and P. F. Fischer, Phys. Rev. E 66:046219 (2002); M. R. Paul, K.-H. Chiam, M. C. Cross, P. F. Fischer, and H. S. Greenside, Physica D (2002), to appear.

[14] H. M. Tufo and P. F. Fischer, in Proc. of the ACM/IEEE SC99 Conf. on High Perfor- 
mance Networking and Computing, IEEE Computer Soc. (CD-ROM format, ADDRESS, 1999), Vol. SC99, ftp://info.mcs.anl.gov/pub/tech_reports/reports/P762.ps.Z.

[15] K. M. S. Bajaj, N. Mukolobwiez, N. Currier, and G. Ahlers, Phys. Rev. Lett. 83, 5282 (1999).

[16] F. Daviaud and A. Pocheau, Europhysics Letters 9, 675 (1989).

[17] F. H. Harlow and J. E. Welch, Phys. Fluids 8, 2182 (1965).

[18] M. Perić, R. Kessler, and G. Scheuerer, Computers and Fluids 16, 389 (1988).

[19] S. W. Armfield, Computers Fluids 29, 1 (1991).

[20] E. Dormy, J. Comp. Physics 151, 676 (1999).

[21] J. V. Kan, SIAM J. Sci. Stat. Comput. 7, 870 (1986).

[22] J. B. Bell, P. Colella, and H. M. Glaz, J. Comp. Phys. 85, 257 (1989).

[23] P. N. Swarztrauber and R. A. Sweet, SIAM J. Num. Anal. 10, 900 (1973).

[24] P. Swarztrauber and R. Sweet, Technical report, NCAR, (unpublished), web documentation: www.scd.ucar.edu/css/software/fishpack. Source code available from www.netlib.org.

[25] E. F. F. Botta et al., Applied Numerical Mathematics 24, 439 (1997).

[26] F. H. Busse, Rep. Prog. Phys. 41, 1929 (1978).

[27] D. Kincaid and W. Cheney, Numerical Analysis, 2nd ed. (Brooks/Cole, Pacific Grove, California, 1996).

[28] A. J. Chorin, Comp. Fluid Mech. 2, 1 (1967).

[29] J. K. Dukowicz and A. S. Dvinsky, J. Comp. Phys. 102, 336 (1992).

[30] J. B. Perot, J. Comp. Phys. 108, 51 (1993). 
[31] S. A. Orszag, M. Israeli, and M. O. Deville, J. Sci. Comput 1, 75 (1986).

[32] P. M. Gresho and R. L. Sani, Int. J. Num. Methods in Fluids 7, 1111 (1987).

[33] S. W. Morris, E. Bodenschatz, D. S. Cannell and G. Ahlers, Phys. Rev. Lett. 71, 2026 (1993).

[34] A. Schlüter, D. Lortz, and F. Busse, J. Fluid Mech. 23, 129 (1965).

[35] R. M. Clever and F. H. Busse, J. Fluid Mech. 65, 625 (1974).

[36] K. R. Kirchartz and J. H. Oertel, J. Fluid Mech. 192, 249 (1988).

[37] D. A. Anderson, J. C. Tannehill, and R. H. Pletcher, Computational Fluid Mechanics and Heat Transfer, (Hemisphere, New York, New York, 1984).

[38] G. P. Gollub, A. R. McCarriar, and J. F. Steinman, J. Fluid Mech. 125, 259 (1982).

[39] H. S. Greenside and W. M. Coughran, Jr., Phys. Rev. A 30, 398 (1984).

[40] R. A. Friesner, L. S. Tuckerman, B. C. Dornblaser, and T. V. Russo, J. Sci. Computing 4, 327 (1989).

[41] M. Hochbruck, C. Lubich, and H. Selhofer, SIAM J. Scientific Computing 19, 1552 (1998).

[42] C. Liu and Z. Liu, J. Comp. Phys. 106, 92 (1993).

[43] M. C. Cross, M. Louie, and D. Meiron, Phys. Rev. E 63, 45201 (2001). 


\section{TABLES}

TABLE I. Estimated order of convergence $p_{h}$ from Eq. (27), as a function of the number of mesh points $N=\Gamma_{x} / h$, for a stationary solution of a two-dimensional square box with periodic sidewalls. The aspect ratio $\Gamma_{x}=2.016$, Rayleigh number $R=1725$, and Prandtl number $\sigma=0.71$. Results are presented for for the temperature field $T(x, z)$ and for the $z$-component of the velocity $u(x, z)$.

\begin{tabular}{c|cc}
\hline \hline$N$ & $p_{h}$ for $T$ & $p_{h}$ for $w$ \\
\hline 16 & 1.46 & 1.43 \\
32 & 1.80 & 1.79 \\
\hline \hline
\end{tabular}

TABLE II. Estimated critical Rayleigh number $\mathrm{R}_{\mathrm{c}}$, based on where the growth rate $\sigma=\sigma(R)$ linearly interpolates through zero. The relative error is defined by $\left(R_{c}-1708\right) / 1708$.

\begin{tabular}{c|cc}
\hline \hline $\mathrm{N}$ & $\mathrm{R}_{\mathrm{c}}$ & Relative error \\
\hline 16 & 1693.0 & 0.9 \\
32 & 1696.6 & 0.7 \\
64 & 1698.5 & 0.5 \\
\hline \hline
\end{tabular}


(a)

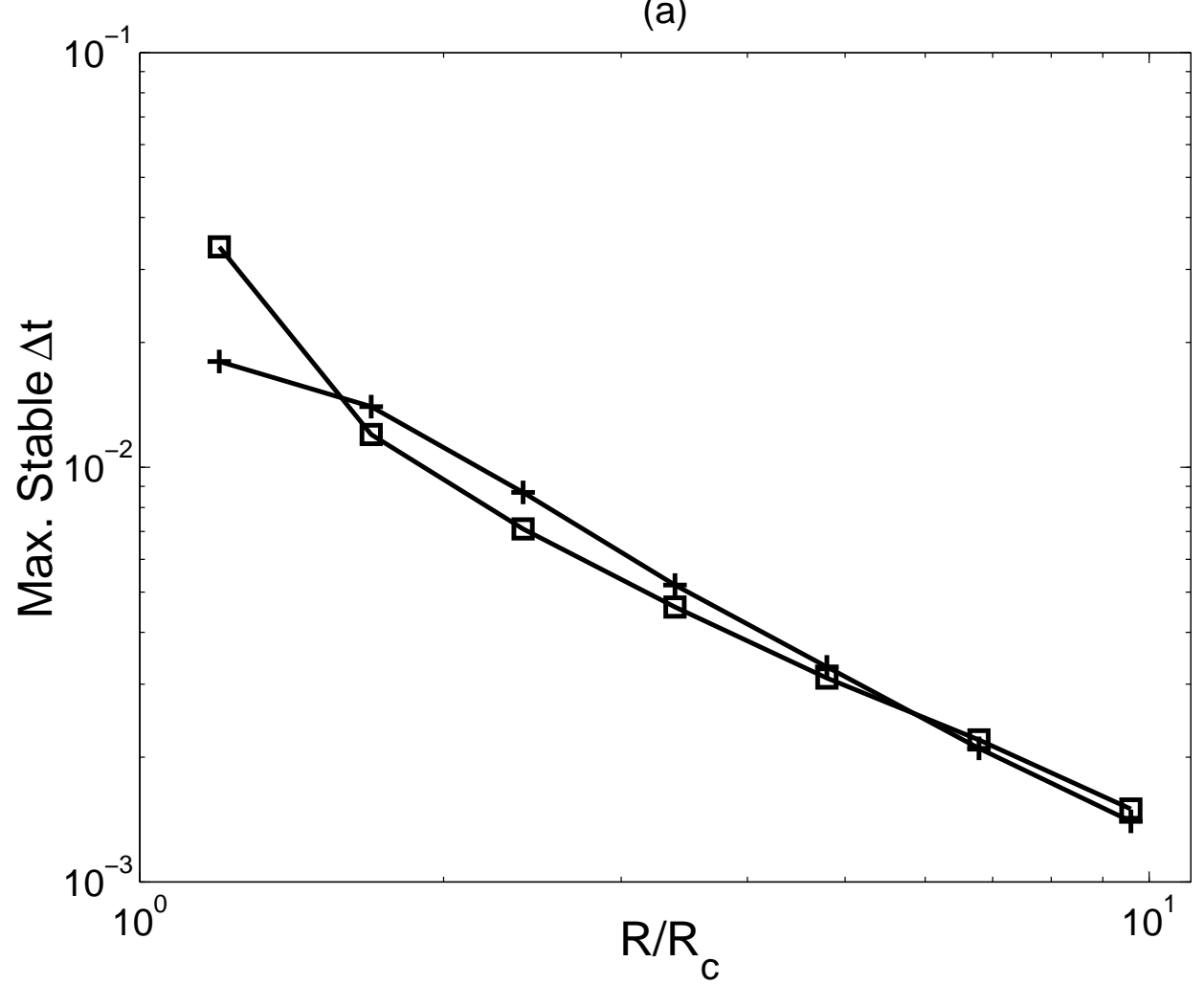

(b)

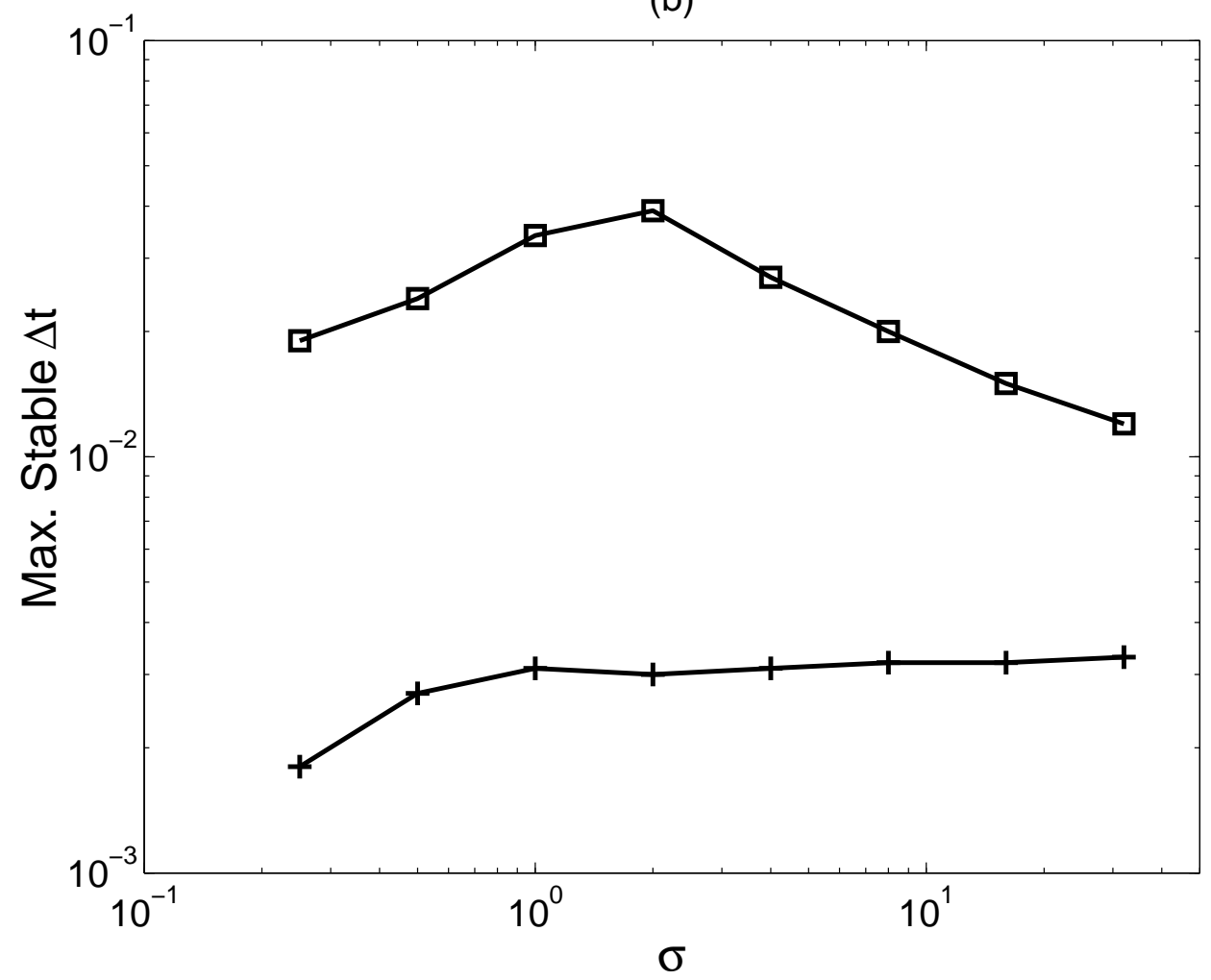

Figure 1. 
(a)

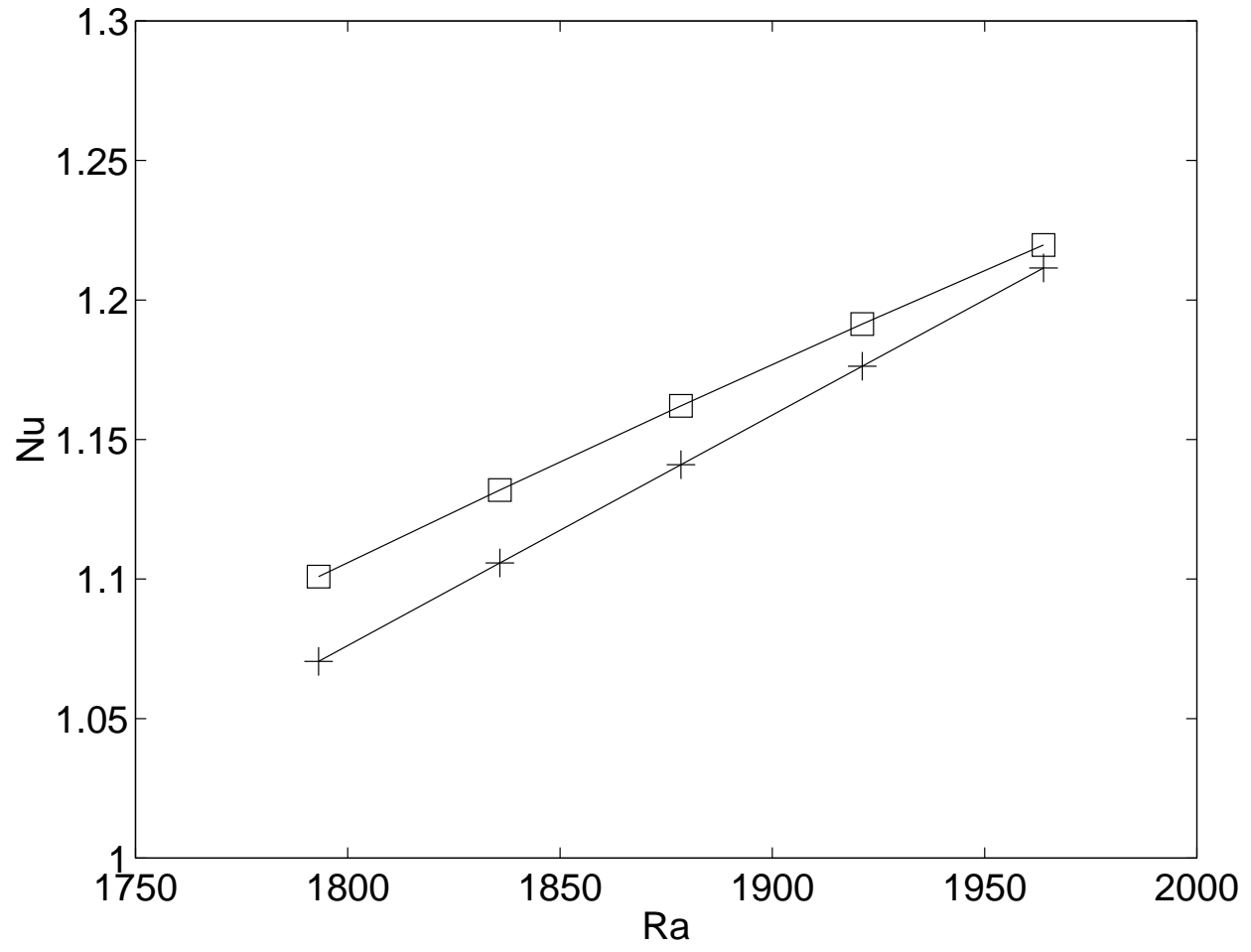

(b)

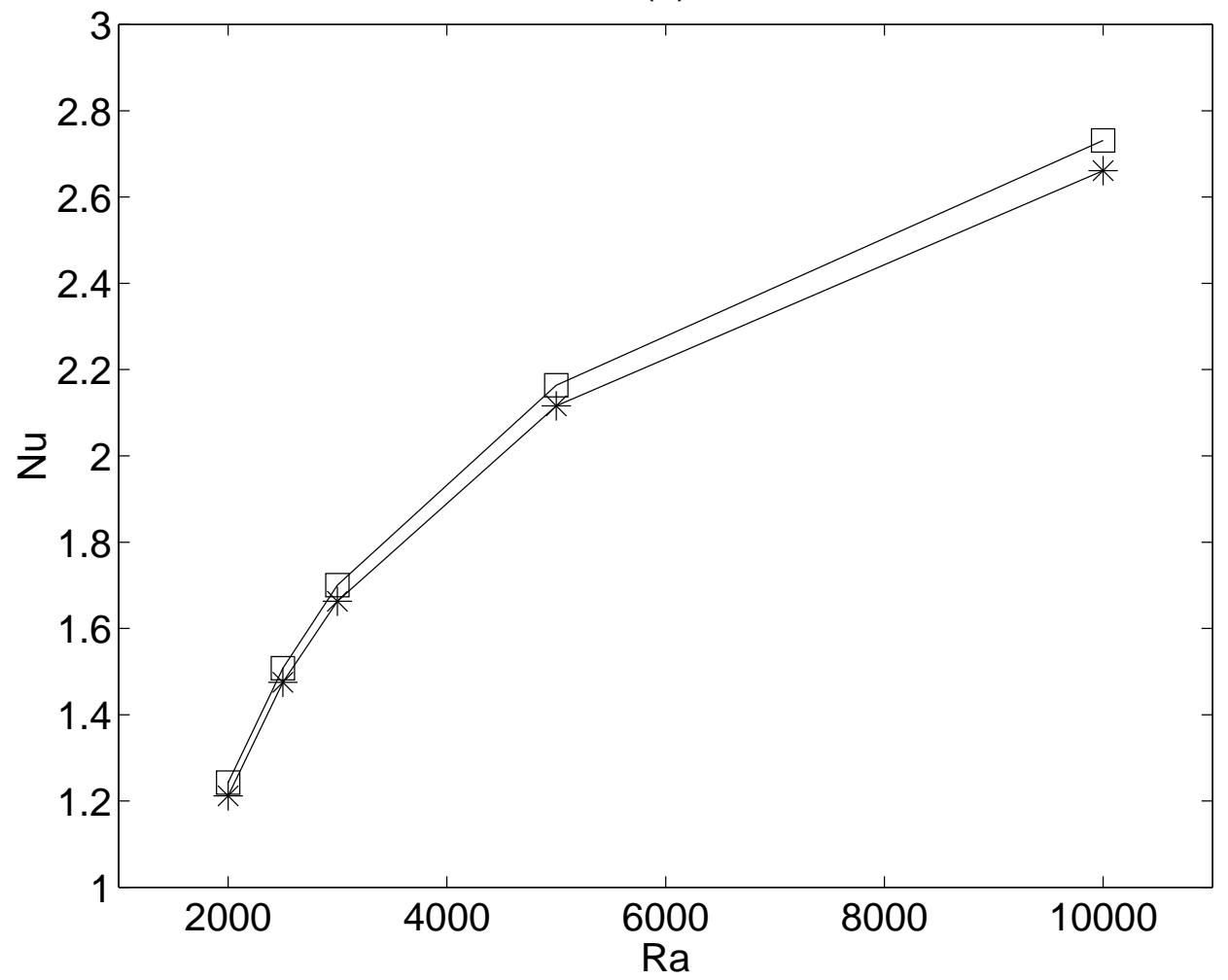

Figure 2. 
(a)

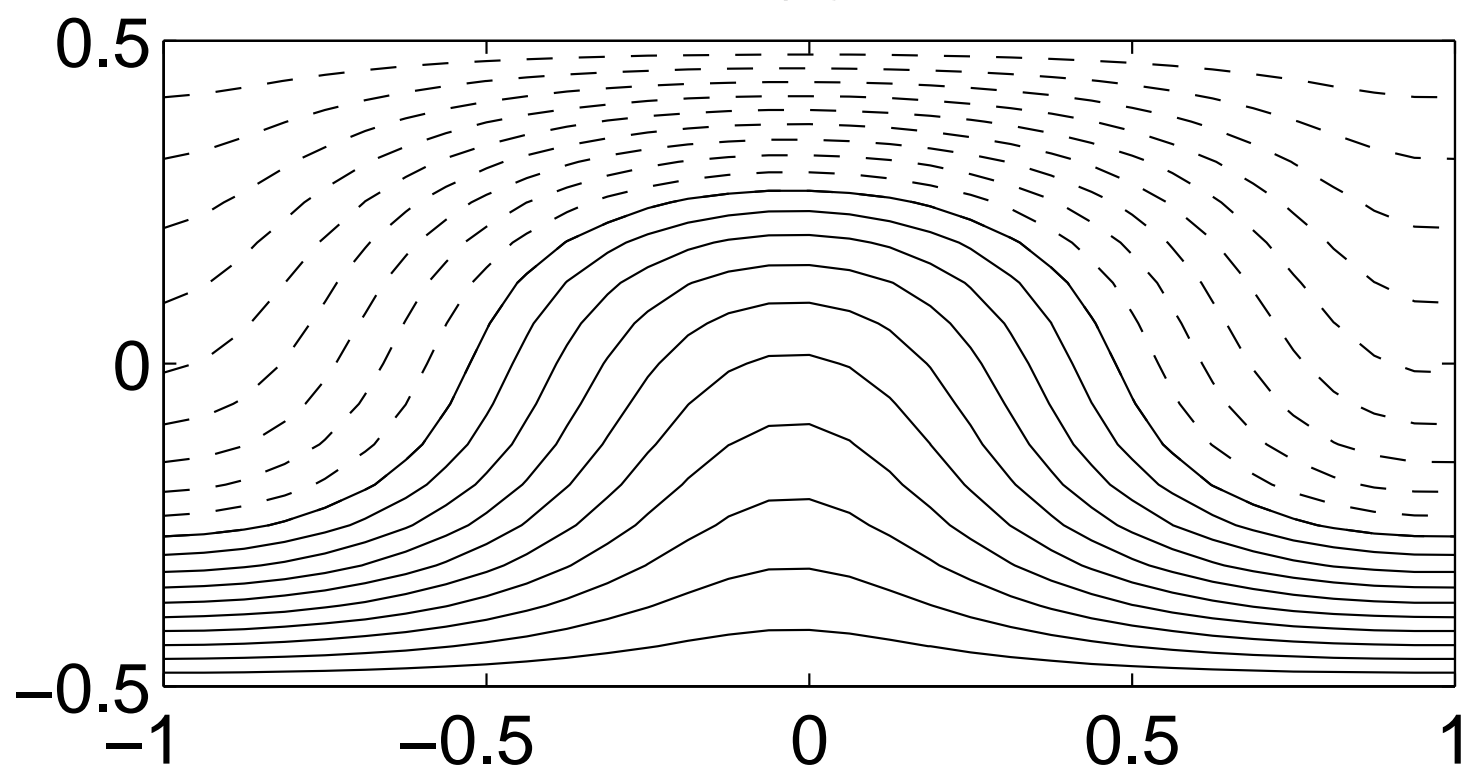

(b)

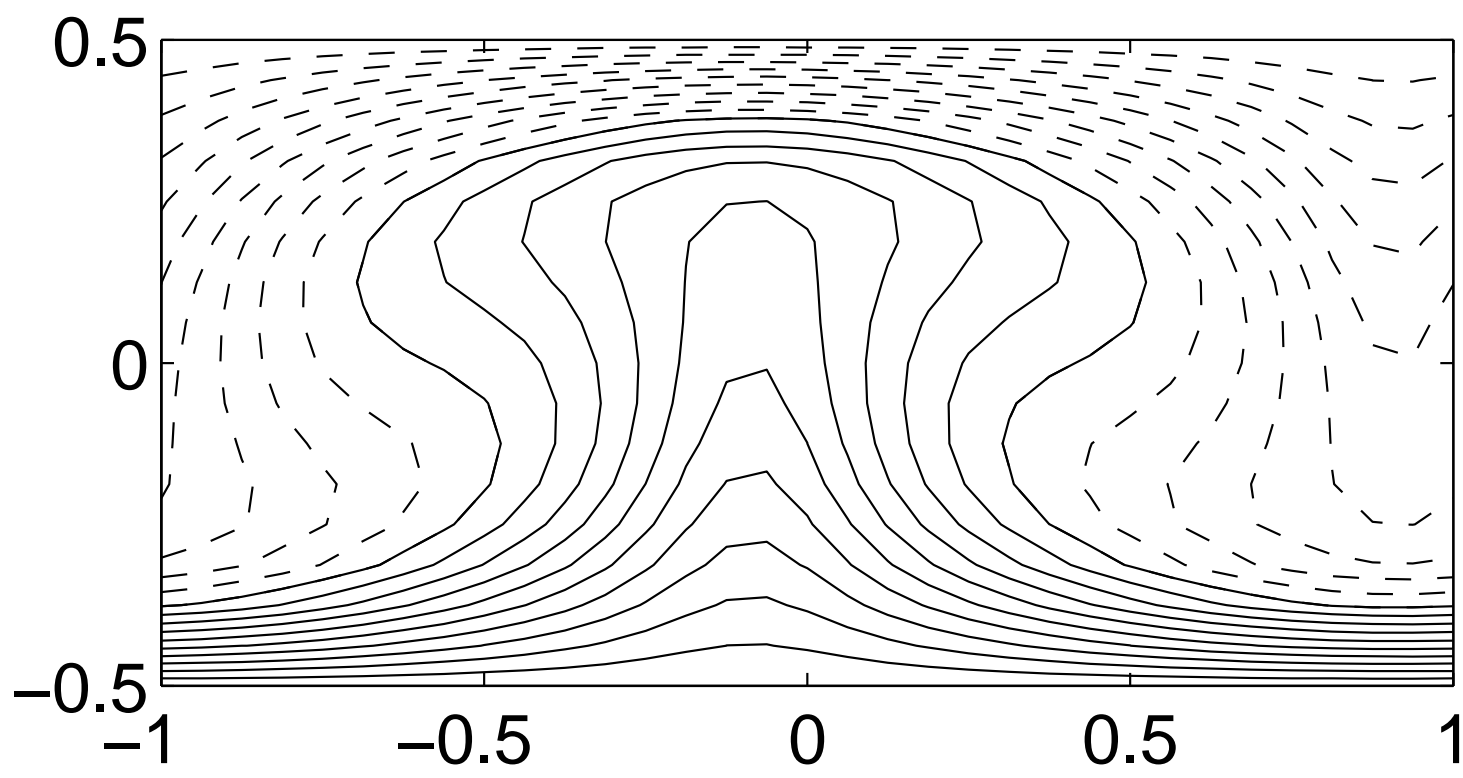

Figure 3. 
(a)

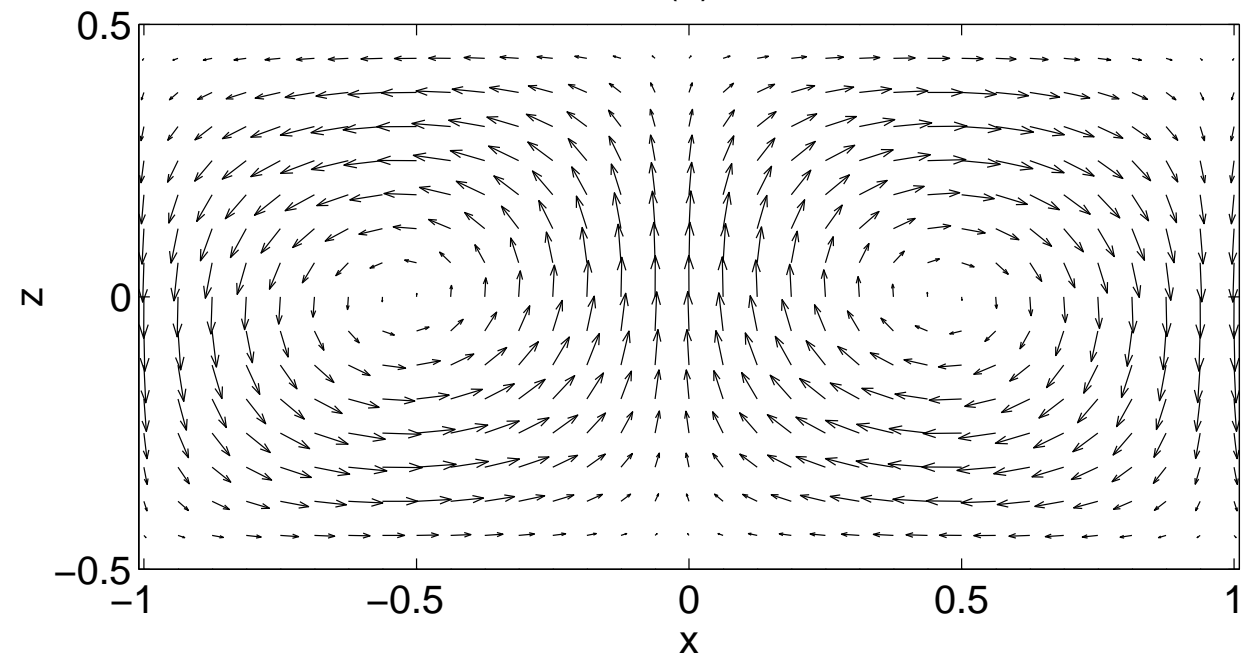

(b)

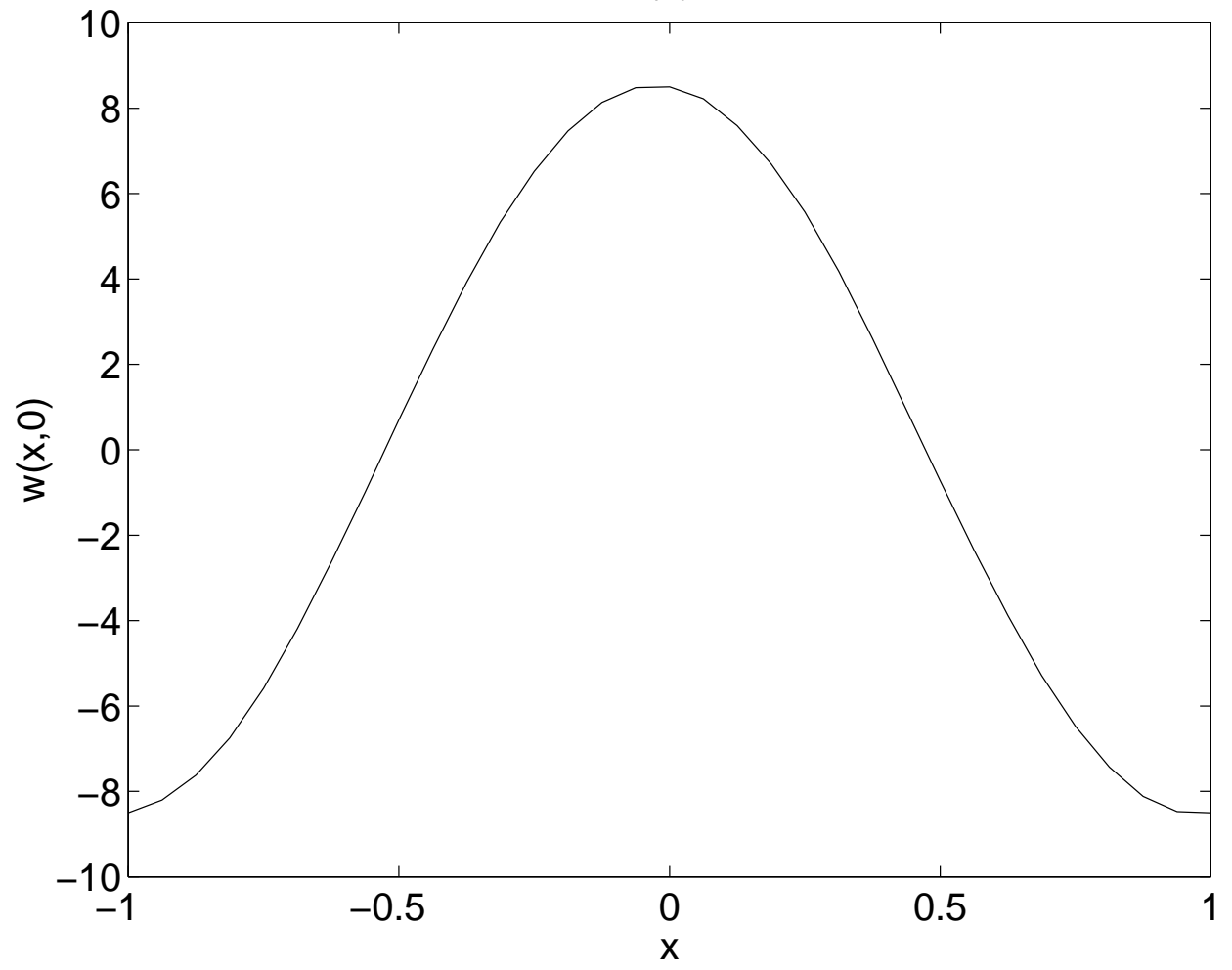

Figures 4. 

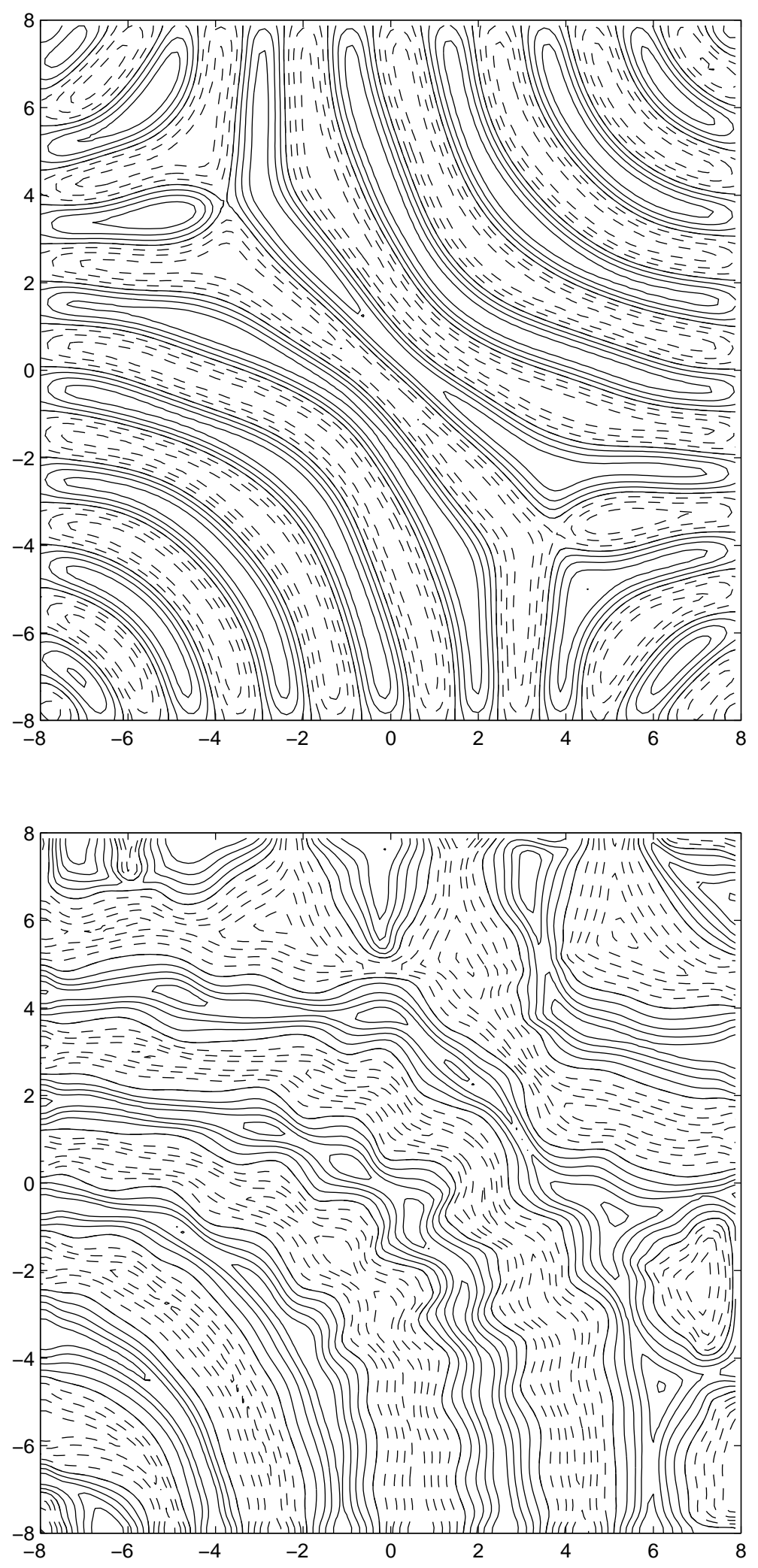

Figure 5. 